\title{
A more accurate half-discrete reverse Hilbert-type inequality with a non-homogeneous kernel
}

Bicheng Yang ${ }^{1 *}$ and Qiang Chen ${ }^{2}$

\section{*Correspondence:}

bcyang@gdei.edu.cn

'Department of Mathematics,

Guangdong University of

Education, Guangzhou, Guangdong

510303, P.R. China

Full list of author information is

available at the end of the article

\begin{abstract}
By means of weight functions and the improved Euler-Maclaurin summation formula, a more accurate half-discrete reverse Hilbert-type inequality with the kernel $\frac{(\min \{1,(x-\gamma)(n-\eta)\})^{\beta}}{\left(\max \{1,(x-\gamma)(n-\eta)\}^{\alpha}\right.}$ and a best constant factor is given. Some equivalent forms, the dual forms as well as some related homogeneous cases, are also considered.
\end{abstract}

MSC: 26D15

Keywords: Hilbert-type inequality; weight function; equivalent form; reverse; dual form

\section{Introduction}

Assuming that $f, g \in L^{2}\left(R_{+}\right),\|f\|=\left\{\int_{0}^{\infty} f^{2}(x) d x\right\}^{\frac{1}{2}}>0,\|g\|>0$, we have the following Hilbert integral inequality $(c f .[1])$ :

$$
\int_{0}^{\infty} \int_{0}^{\infty} \frac{f(x) g(y)}{x+y} d x d y<\pi\|f\|\|g\|
$$

where the constant factor $\pi$ is best possible. If $a=\left\{a_{n}\right\}_{n=1}^{\infty}, b=\left\{b_{n}\right\}_{n=1}^{\infty} \in l^{2},\|a\|=$ $\left\{\sum_{n=1}^{\infty} a_{n}^{2}\right\}^{\frac{1}{2}}>0,\|b\|>0$, then we have the following analogous discrete Hilbert inequality:

$$
\sum_{m=1}^{\infty} \sum_{n=1}^{\infty} \frac{a_{m} b_{n}}{m+n}<\pi\|a\|\|b\|
$$

with the same best constant factor $\pi$. Inequalities (1) and (2) are important in analysis and its applications (cf. [2-4]).

In 1998, by introducing an independent parameter $\lambda \in(0,1]$, Yang [5] gave an extension of (1). For generalizing the results from [5], Yang [6] gave some best extensions of (1) and (2): If $p>1, \frac{1}{p}+\frac{1}{q}=1, \lambda_{1}+\lambda_{2}=\lambda, k_{\lambda}(x, y)$ is a non-negative homogeneous function of degree $-\lambda$ satisfying $k\left(\lambda_{1}\right)=\int_{0}^{\infty} k_{\lambda}(t, 1) t^{\lambda_{1}-1} d t \in R_{+}, \phi(x)=x^{p\left(1-\lambda_{1}\right)-1}, \psi(x)=x^{q\left(1-\lambda_{2}\right)-1}, f(\geq 0) \in$ $L_{p, \phi}\left(R_{+}\right)=\left\{f \mid\|f\|_{p, \phi}:=\left\{\int_{0}^{\infty} \phi(x)|f(x)|^{p} d x\right\}^{\frac{1}{p}}<\infty\right\}, g(\geq 0) \in L_{q, \psi}\left(R_{+}\right)$, and $\|f\|_{p, \phi},\|g\|_{q, \psi}>$ 0 , then

$$
\int_{0}^{\infty} \int_{0}^{\infty} k_{\lambda}(x, y) f(x) g(y) d x d y<k\left(\lambda_{1}\right)\|f\|_{p, \phi}\|g\|_{q, \psi},
$$

@2014 Yang and Chen; licensee Springer. This is an Open Access article distributed under the terms of the Creative Commons Attribution License (http://creativecommons.org/licenses/by/2.0), which permits unrestricted use, distribution, and reproduction in any medium, provided the original work is properly cited. 
where the constant factor $k\left(\lambda_{1}\right)$ is best possible. Moreover if the value of $k_{\lambda}(x, y)$ is finite and $k_{\lambda}(x, y) x^{\lambda_{1}-1}\left(k_{\lambda}(x, y) y^{\lambda_{2}-1}\right)$ is decreasing for $x>0(y>0)$, then for $a_{m}, b_{n} \geq 0, a=\left\{a_{m}\right\}_{m=1}^{\infty} \in$ $l_{p, \phi}=\left\{a \mid\|a\|_{p, \phi}:=\left\{\sum_{n=1}^{\infty} \phi(n)\left|a_{n}\right|^{p}\right\}^{\frac{1}{p}}<\infty\right\}$, and $b=\left\{b_{n}\right\}_{n=1}^{\infty} \in l_{q, \psi},\|a\|_{p, \phi},\|b\|_{q, \psi}>0$, we have

$$
\sum_{m=1}^{\infty} \sum_{n=1}^{\infty} k_{\lambda}(m, n) a_{m} b_{n}<k\left(\lambda_{1}\right)\|a\|_{p, \phi}\|b\|_{q, \psi}
$$

where the constant $k\left(\lambda_{1}\right)$ is still best value. Clearly, for $p=q=2, \lambda=1, k_{1}(x, y)=\frac{1}{x+y}, \lambda_{1}=$ $\lambda_{2}=\frac{1}{2}$, (3) reduces to (1), while (4) reduces to (2). The reverses of (3) and (4) as well as the equivalent forms are also considered by [6].

Some other results about integral and discrete Hilbert-type inequalities can be found in [7-15]. On half-discrete Hilbert-type inequalities with the general non-homogeneous kernels, Hardy et al. provided a few results in Theorem 351 of [1]. But they did not prove that the constant factors are best possible. In 2005, Yang [16] gave a result with the kernel $\frac{1}{(1+n x)^{\lambda}}$ by introducing a variable and proved that the constant factor is best possible. Very recently, Yang [17] and [18] gave the following half-discrete reverse Hilbert inequality with best constant factor: For $0<p<1, \frac{1}{p}+\frac{1}{q}=1, \lambda_{1}>0,0<\lambda_{2} \leq 1, \lambda_{1}+\lambda_{2}=\lambda, \theta_{\lambda}(x)=O\left(\frac{1}{x^{\lambda_{2}}}\right) \in$ $(0,1), \widetilde{\phi}(x)=\left(1-\theta_{\lambda}(x)\right) x^{p\left(1-\lambda_{1}\right)-1}$,

$$
\int_{0}^{\infty} f(x) \sum_{n=1}^{\infty} \frac{a_{n}}{(x+n)^{\lambda}} d x>B\left(\lambda_{1}, \lambda_{2}\right)\|f\|_{p, \tilde{\phi}}\|a\|_{q, \psi} .
$$

In this paper, by means of weight functions and the improved Euler-Maclaurin summation formula, a more accurate half-discrete reverse Hilbert-type inequality with the kernel $\frac{(\min \{1,(x-\gamma)(n-\eta)\}\}^{\beta}}{(\max \{1,(x-\gamma)(n-\eta)\})^{\alpha}}$ similar to (5) and a best constant factor is given. Moreover, some equivalent forms, the dual forms as well as some relating homogeneous cases are also considered.

\section{Some lemmas}

Lemma 1 If $n_{0} \in \mathbf{N}, s>n_{0}, g_{1}(y)\left(y \in\left[n_{0}, s\right)\right), g_{2}(y)(y \in[s, \infty))$ are continuous decreasing functions satisfying $g_{1}\left(n_{0}\right)-g_{1}(s-0)+g_{2}(s)>0, g_{2}(\infty)=0$, define a function $g(y)$ as follows:

$$
g(y):= \begin{cases}g_{1}(y), & y \in\left[n_{0}, s\right) \\ g_{2}(y), & y \in[s, \infty)\end{cases}
$$

Then there exists $\varepsilon \in[0,1]$, such that

$$
\begin{aligned}
& \frac{-1}{8}\left[g_{1}\left(n_{0}\right)+\varepsilon\left(g_{2}(s)-g_{1}(s-0)\right)\right] \\
& <\int_{n_{0}}^{\infty} \rho(y) g(y) d y<\frac{1-\varepsilon}{8}\left(g_{2}(s)-g_{1}(s-0)\right),
\end{aligned}
$$

where $\rho(y)=y-[y]-\frac{1}{2}$ is the Bernoulli function of the first order. In particular, for $g_{1}(y)=0$, $y \in\left[n_{0}, s\right)$, we have $g_{2}(s)>0$ and

$$
\frac{-1}{8} g_{2}(s)<\int_{s}^{\infty} \rho(y) g(y) d y<\frac{1}{8} g_{2}(s)
$$


for $g_{2}(y)=0, y \in[s, \infty)$, if $g_{1}(s-0) \geq 0$, then it follows $g_{1}\left(n_{0}\right)>0$ and

$$
\frac{-1}{8} g_{1}\left(n_{0}\right)<\int_{n_{0}}^{s} \rho(y) g_{1}(y) d y<0 .
$$

Proof Define a continuous decreasing function $\widetilde{g}(y)$ as follows:

$$
\widetilde{g}(y):= \begin{cases}g_{1}(y)+g_{2}(s)-g_{1}(s-0), & y \in\left[n_{0}, s\right), \\ g_{2}(y), & y \in[s, \infty) .\end{cases}
$$

Then it follows that

$$
\begin{aligned}
\int_{n_{0}}^{\infty} \rho(y) g(y) d y & =\int_{n_{0}}^{s} \rho(y) g(y) d y+\int_{s}^{\infty} \rho(y) g(y) d y \\
& =\int_{n_{0}}^{s} \rho(y)\left(\widetilde{g}(y)-g_{2}(s)+g_{1}(s-0)\right) d y+\int_{s}^{\infty} \rho(y) \widetilde{g}(y) d y \\
& =\int_{n_{0}}^{\infty} \rho(y) \widetilde{g}(y) d y-\left(g_{2}(s)-g_{1}(s-0)\right) \int_{n_{0}}^{s} \rho(y) d y, \\
\int_{n_{0}}^{s} \rho(y) d y= & \int_{n_{0}}^{[s]} \rho(y) d y+\int_{[s]}^{s} \rho(y) d y=\int_{[s]}^{s}\left(y-[s]-\frac{1}{2}\right) d y \\
= & \frac{1}{8}\left[4\left(s-[s]-\frac{1}{2}\right)^{2}-1\right]=\frac{\varepsilon-1}{8} \quad(\varepsilon \in[0,1]) .
\end{aligned}
$$

Since $\widetilde{g}\left(n_{0}\right)=g_{1}\left(n_{0}\right)+g_{2}(s)-g_{1}(s-0)>0, \tilde{g}(y)$ is a non-constant continuous decreasing function with $\widetilde{g}(\infty)=g_{2}(\infty)=0$, by the improved Euler-Maclaurin summation formula (cf. [6], Theorem 2.2.2), it follows that

$$
\frac{-1}{8}\left(g_{1}\left(n_{0}\right)+g_{2}(s)-g_{1}(s-0)\right)=\frac{-1}{8} \widetilde{g}\left(n_{0}\right)<\int_{n_{0}}^{\infty} \rho(y) \widetilde{g}(y) d y<0,
$$

and then in view of the above results and by simple calculation, we have (7).

Lemma 2 If $0<\alpha+\beta \leq 2, \gamma \in \mathbf{R}, \eta \leq 1-\frac{\alpha+\beta}{8}\left(1+\sqrt{3+\frac{4}{\alpha+\beta}}\right)$, and $\omega(n)$ and $\varpi(x)$ are weight functions given by

$$
\begin{aligned}
& \omega(n):=\int_{\gamma}^{\infty} \frac{(\min \{1,(x-\gamma)(n-\eta)\})^{\beta}}{(\max \{1,(x-\gamma)(n-\eta)\})^{\alpha}} \frac{(n-\eta)^{\frac{\alpha-\beta}{2}}}{(x-\gamma)^{1-\frac{\alpha-\beta}{2}}} d x, \quad n \in \mathbf{N}, \\
& \varpi(x):=\sum_{n=1}^{\infty} \frac{(\min \{1,(x-\gamma)(n-\eta)\})^{\beta}}{(\max \{1,(x-\gamma)(n-\eta)\})^{\alpha}} \frac{(x-\gamma)^{\frac{\alpha-\beta}{2}}}{(n-\eta)^{1-\frac{\alpha-\beta}{2}}}, \quad x>\gamma,
\end{aligned}
$$

then we have

$$
\begin{aligned}
& 0<\frac{4}{\alpha+\beta}(1-\theta(x))<\varpi(x)<\omega(n)=\frac{4}{\alpha+\beta}, \\
& \theta(x)= \begin{cases}\frac{1}{2}(1-\eta)^{\frac{\alpha+\beta}{2}}(x-\gamma)^{\frac{\alpha+\beta}{2}}, & 0<x-\gamma \leq \frac{1}{1-\eta}, \\
1-\frac{1}{2}(1-\eta)^{-\frac{\alpha+\beta}{2}}(x-\gamma)^{-\frac{\alpha+\beta}{2}}, & x-\gamma>\frac{1}{1-\eta} .\end{cases}
\end{aligned}
$$


Proof Substituting $t=(x-\gamma)(n-\eta)$ in (10), and by simple calculation, we have

$$
\omega(n)=\int_{0}^{\infty} \frac{(\min \{1, t\})^{\beta}}{(\max \{1, t\})^{\alpha}} t^{\frac{\alpha-\beta}{2}-1} d t=\int_{0}^{1} t^{\beta+\frac{\alpha-\beta}{2}-1} d t+\int_{1}^{\infty} t^{-\alpha+\frac{\alpha-\beta}{2}-1} d t=\frac{4}{\alpha+\beta} .
$$

For fixed $x>\gamma$, we find

$$
\begin{aligned}
& h(x, y):=(x-\gamma)^{\frac{\alpha-\beta}{2}} \frac{(\min \{1,(x-\gamma)(y-\eta)\})^{\beta}}{(\max \{1,(x-\gamma)(y-\eta)\})^{\alpha}}(y-\eta)^{\frac{\alpha-\beta}{2}-1} \\
&= \begin{cases}(x-\gamma)^{\frac{\alpha+\beta}{2}}(y-\eta)^{\frac{\alpha+\beta}{2}-1}, & \eta<y<\eta+\frac{1}{x-\gamma}, \\
(x-\gamma)^{-\frac{\alpha+\beta}{2}}(y-\eta)^{-\frac{\alpha+\beta}{2}}-1, & y \geq \eta+\frac{1}{x-\gamma},\end{cases} \\
& h_{y}^{\prime}(x, y)= \begin{cases}-\left(1-\frac{\alpha+\beta}{2}\right)(x-\gamma)^{\frac{\alpha+\beta}{2}}(y-\eta)^{\frac{\alpha+\beta}{2}-2}, & \eta<y<\eta+\frac{1}{x-\gamma}, \\
-\left(\frac{\alpha+\beta}{2}+1\right)(x-\gamma)^{-\frac{\alpha+\beta}{2}}(y-\eta)^{-\frac{\alpha+\beta}{2}-2}, & y \geq \eta+\frac{1}{x-\gamma},\end{cases} \\
& \int_{\eta}^{\infty} h(x, y) d y \stackrel{t=(x-\gamma)(y-\eta)}{=} \int_{0}^{\infty} \frac{(\min \{1, t\})^{\beta}}{(\max \{1, t\})^{\alpha}} t^{\frac{\alpha-\beta}{2}-1} d t=\frac{4}{\alpha+\beta} .
\end{aligned}
$$

By the Euler-Maclaurin summation formula (cf. [6]), it follows that

$$
\begin{aligned}
\varpi(x) & =\sum_{n=1}^{\infty} h(x, n)=\int_{1}^{\infty} h(x, y) d y+\frac{1}{2} h(x, 1)+\int_{1}^{\infty} \rho(y) h_{y}^{\prime}(x, y) d y \\
& =\int_{\eta}^{\infty} h(x, y) d y-R(x)=\frac{4}{\alpha+\beta}-R(x), \\
R(x) & :=\int_{\eta}^{1} h(x, y) d y-\frac{1}{2} h(x, 1)-\int_{1}^{\infty} \rho(y) h_{y}^{\prime}(x, y) d y .
\end{aligned}
$$

(i) For $0<x-\gamma<\frac{1}{1-\eta}$, we obtain $-\frac{1}{2} h(x, 1)=-\frac{1}{2}(x-\gamma)^{\frac{\alpha+\beta}{2}}(1-\eta)^{\frac{\alpha+\beta}{2}-1}$, and

$$
\int_{\eta}^{1} h(x, y) d y=(x-\gamma)^{\frac{\alpha+\beta}{2}} \int_{\eta}^{1}(y-\eta)^{\frac{\alpha+\beta}{2}-1} d y=\frac{2(1-\eta)^{\frac{\alpha+\beta}{2}}}{\alpha+\beta}(x-\gamma)^{\frac{\alpha+\beta}{2}} .
$$

Setting $g(y):=-h_{y}^{\prime}(x, y)$, wherefrom $g_{1}(y)=\left(1-\frac{\alpha+\beta}{2}\right)(x-\gamma)^{\frac{\alpha+\beta}{2}}(y-\eta)^{\frac{\alpha+\beta}{2}-2}, g_{2}(y)=\left(\frac{\alpha+\beta}{2}+\right.$ 1) $(x-\gamma)^{-\frac{\alpha+\beta}{2}}(y-\eta)^{-\frac{\alpha+\beta}{2}-2}$ and

$$
\begin{aligned}
& g_{2}\left(\eta+\frac{1}{x-\gamma}\right)-g_{1}\left(\left(\eta+\frac{1}{x-\gamma}\right)-0\right) \\
& \quad=\left(\frac{\alpha+\beta}{2}+1\right)(x-\gamma)^{2}-\left(1-\frac{\alpha+\beta}{2}\right)(x-\gamma)^{2} \\
& \quad=(\alpha+\beta)(x-\gamma)^{2}>0,
\end{aligned}
$$

then by (7), we find

$$
\begin{aligned}
-\int_{1}^{\infty} \rho(y) h_{y}^{\prime}(x, y) d y & =\int_{1}^{\infty} \rho(y) g(y) d y \\
& >\frac{-1}{8}\left[g_{1}(1)+g_{2}\left(\eta+\frac{1}{x-\gamma}\right)-g_{1}\left(\left(\eta+\frac{1}{x-\gamma}\right)-0\right)\right]
\end{aligned}
$$




$$
\begin{aligned}
= & \frac{-1}{8}\left[\left(1-\frac{\alpha+\beta}{2}\right)(x-\gamma)^{\frac{\alpha+\beta}{2}}(1-\eta)^{\frac{\alpha+\beta}{2}-2}+(\alpha+\beta)(x-\gamma)^{2}\right] \\
> & \frac{-1}{8}\left[\left(1-\frac{\alpha+\beta}{2}\right)(1-\eta)^{\frac{\alpha+\beta}{2}-2}(x-\gamma)^{\frac{\alpha+\beta}{2}}\right. \\
& \left.+(\alpha+\beta)(1-\eta)^{\frac{\alpha+\beta}{2}-2}(x-\gamma)^{\frac{\alpha+\beta}{2}-2}(x-\gamma)^{2}\right] \\
= & \frac{-1}{8}\left(1+\frac{\alpha+\beta}{2}\right)(1-\eta)^{\frac{\alpha+\beta}{2}-2}(x-\gamma)^{\frac{\alpha+\beta}{2}} .
\end{aligned}
$$

In view of (11) and the above results, since for $\eta \leq 1-\frac{\alpha+\beta}{8}\left(1+\sqrt{3+\frac{4}{\alpha+\beta}}\right)$, namely $1-\eta \geq$ $\frac{\alpha+\beta}{8}\left(1+\sqrt{3+\frac{4}{\alpha+\beta}}\right)$, it follows that

$$
\begin{aligned}
R(x)> & \frac{2}{\alpha+\beta}(1-\eta)^{\frac{\alpha+\beta}{2}}(x-\gamma)^{\frac{\alpha+\beta}{2}}-\frac{1}{2}(x-\gamma)^{\frac{\alpha+\beta}{2}}(1-\eta)^{\frac{\alpha+\beta}{2}-1} \\
& -\frac{1}{8}\left(1+\frac{\alpha+\beta}{2}\right)(1-\eta)^{\frac{\alpha+\beta}{2}-2}(x-\gamma)^{\frac{\alpha+\beta}{2}} \\
= & {\left[\frac{2(1-\eta)^{2}}{\alpha+\beta}-\frac{(1-\eta)}{2}-\frac{2+\alpha+\beta}{16}\right] \frac{(x-\gamma)^{\frac{\alpha+\beta}{2}}}{(1-\eta)^{2-\frac{\alpha+\beta}{2}}} \geq 0 . }
\end{aligned}
$$

(ii) For $x-\gamma \geq \frac{1}{1-\eta}$, we obtain $-\frac{1}{2} h(x, 1)=-\frac{1}{2}(x-\gamma)^{-\frac{\alpha+\beta}{2}}(1-\eta)^{-\frac{\alpha+\beta}{2}-1}$, and

$$
\begin{aligned}
\int_{\eta}^{1} h(x, y) d y & =\int_{\eta}^{\eta+\frac{1}{x-\gamma}} \frac{(x-\gamma)^{\frac{\alpha+\beta}{2}}}{(y-\eta)^{1-\frac{\alpha+\beta}{2}}} d y+\int_{\eta+\frac{1}{x-\gamma}}^{1} \frac{(x-\gamma)^{-\frac{\alpha+\beta}{2}}}{(y-\eta)^{\frac{\alpha+\beta}{2}+1}} d y \\
& =\frac{4}{\alpha+\beta}-\frac{2}{\alpha+\beta}(1-\eta)^{-\frac{\alpha+\beta}{2}}(x-\gamma)^{-\frac{\alpha+\beta}{2}} \\
& \geq \frac{4(1-\eta)^{-\frac{\alpha+\beta}{2}}}{\alpha+\beta}(x-\gamma)^{-\frac{\alpha+\beta}{2}}-\frac{2(1-\eta)^{-\frac{\alpha+\beta}{2}}}{\alpha+\beta}(x-\gamma)^{-\frac{\alpha+\beta}{2}} \\
& =\frac{2}{\alpha+\beta}(1-\eta)^{-\frac{\alpha+\beta}{2}}(x-\gamma)^{-\frac{\alpha+\beta}{2}}
\end{aligned}
$$

Since for $y \geq 1, y-\eta \geq \frac{1}{x-\gamma}$, by the improved Euler-Maclaurin summation formula (cf. [6]), it follows that

$$
\begin{aligned}
-\int_{1}^{\infty} \rho(y) h_{y}^{\prime}(x, y) d y & =\left(\frac{\alpha+\beta}{2}+1\right)(x-\gamma)^{-\frac{\alpha+\beta}{2}} \int_{1}^{\infty} \rho(y)(y-\eta)^{-\frac{\alpha+\beta}{2}-2} d y \\
& >-\frac{1}{8}\left(\frac{\alpha+\beta}{2}+1\right)(x-\gamma)^{-\frac{\alpha+\beta}{2}}(1-\eta)^{-\frac{\alpha+\beta}{2}-2} .
\end{aligned}
$$

In view of (13) and the above results, for $1-\eta \geq \frac{\alpha+\beta}{8}\left(1+\sqrt{3+\frac{4}{\alpha+\beta}}\right)$, we find

$$
\begin{aligned}
R(x)> & \frac{2}{\alpha+\beta}(1-\eta)^{-\frac{\alpha+\beta}{2}}(x-\gamma)^{-\frac{\alpha+\beta}{2}}-\frac{1}{2}(1-\eta)^{-\frac{\alpha+\beta}{2}-1}(x-\gamma)^{-\frac{\alpha+\beta}{2}} \\
& -\frac{1}{8}\left(\frac{\alpha+\beta}{2}+1\right)(1-\eta)^{-\frac{\alpha+\beta}{2}-2}(x-\gamma)^{-\frac{\alpha+\beta}{2}} \\
= & {\left[\frac{2(1-\eta)^{2}}{\alpha+\beta}-\frac{1-\eta}{2}-\frac{2+\alpha+\beta}{16}\right] \frac{(x-\gamma)^{-\frac{\alpha+\beta}{2}}}{(1-\eta)^{2+\frac{\alpha+\beta}{2}}} \geq 0 . }
\end{aligned}
$$


Hence for $x>\gamma$, we have $R(x)>0$, and then $\varpi(x)<\omega(n)=\frac{4}{\alpha+\beta}$.

On the other-hand, since $h(x, y)$ is decreasing with respect to $y>\eta$, we find

$$
\begin{gathered}
\varpi(x)>\int_{1}^{\infty} \frac{(\min \{1,(x-\gamma)(y-\eta)\})^{\beta}}{(\max \{1,(x-\gamma)(y-\eta)\})^{\alpha}} \frac{(x-\gamma)^{\frac{\alpha-\beta}{2}}}{(y-\eta)^{1-\frac{\alpha-\beta}{2}}} d y \\
\stackrel{t=(x-\gamma)(y-\eta)}{=} \int_{(1-\eta)(x-\gamma)}^{\infty} \frac{(\min \{1, t\})^{\beta}}{(\max \{1, t\})^{\alpha}} t^{\frac{\alpha-\beta}{2}-1} d t=\frac{4}{\alpha+\beta}(1-\theta(x)),
\end{gathered}
$$

where $\theta(x):=\frac{\alpha+\beta}{4} \int_{0}^{(1-\eta)(x-\gamma)} \frac{(\min \{1, t)\}^{\beta}}{\left(\max \{1, t)^{\alpha}\right.} t^{\frac{\alpha-\beta}{2}}-1 d t \in(0,1)$.

(i) For $0<x-\gamma \leq \frac{1}{1-\eta}$, we obtain

$$
\theta(x)=\frac{\alpha+\beta}{4} \int_{0}^{(1-\eta)(x-\gamma)} t^{\beta+\frac{\alpha-\beta}{2}-1} d t=\frac{1}{2}(1-\eta)^{\frac{\alpha+\beta}{2}}(x-\gamma)^{\frac{\alpha+\beta}{2}} .
$$

(ii) For $x-\gamma>\frac{1}{1-\eta}$, it follows that

$$
\begin{aligned}
\theta(x) & =\frac{\alpha+\beta}{4}\left[\int_{0}^{1} t^{\beta+\frac{\alpha-\beta}{2}-1} d t+\int_{1}^{(1-\eta)(x-\gamma)} t^{-\alpha+\frac{\alpha-\beta}{2}-1} d t\right] \\
& =1-\frac{1}{2}(1-\eta)^{-\frac{\alpha+\beta}{2}}(x-\gamma)^{-\frac{\alpha+\beta}{2}} .
\end{aligned}
$$

Hence we have (11) and (12).

Lemma 3 Let the assumptions of Lemma 2 be fulfilled and additionally, let $0<p<1$ or $p<0, \frac{1}{p}+\frac{1}{q}=1, a_{n} \geq 0, n \in \mathbf{N}, f(x)$ be a non-negative measurable function in $(\gamma, \infty)$. Then we have the following inequalities:

$$
\begin{aligned}
J & :=\left\{\sum_{n=1}^{\infty}(n-\beta)^{\frac{p(\alpha-\beta)}{2}-1}\left[\int_{\gamma}^{\infty} \frac{(\min \{1,(x-\gamma)(n-\eta)\})^{\beta}}{(\max \{1,(x-\gamma)(n-\eta)\})^{\alpha}} f(x) d x\right]^{p}\right\}^{\frac{1}{p}} \\
& \geq\left(\frac{4}{\alpha+\beta}\right)^{\frac{1}{q}}\left\{\int_{\gamma}^{\infty} \varpi(x)(x-\gamma)^{p\left(1-\frac{\alpha-\beta}{2}\right)-1} f^{p}(x) d x\right\}^{\frac{1}{p}}, \\
L_{1} & :=\left\{\int_{\gamma}^{\infty} \frac{(x-\alpha)^{\frac{q(\alpha-\beta)}{2}}-1}{[\varpi(x)]^{q-1}}\left[\sum_{n=1}^{\infty} \frac{(\min \{1,(x-\gamma)(n-\eta)\})^{\beta} a_{n}}{(\max \{1,(x-\gamma)(n-\eta)\})^{\alpha}}\right]^{q} d x\right\}^{\frac{1}{q}} \\
& \geq\left\{\frac{4}{\alpha+\beta} \sum_{n=1}^{\infty}(n-\eta)^{q\left(1-\frac{\alpha-\beta}{2}\right)-1} a_{n}^{q}\right\}^{\frac{1}{q}} .
\end{aligned}
$$

Proof For $0<p<1$, setting $k(x, n):=\frac{(\min \{1,(x-\gamma)(n-\eta)\})^{\beta}}{(\max \{1,(x-\gamma)(n-\eta)\})^{\alpha}}$, by the reverse Hölder inequality $(c f$. [19]) and (11), it follows that

$$
\begin{aligned}
& {\left[\int_{\gamma}^{\infty} \frac{(\min \{1,(x-\gamma)(n-\eta)\})^{\beta}}{(\max \{1,(x-\gamma)(n-\eta)\})^{\alpha}} f(x) d x\right]^{p}} \\
& \quad=\left\{\int_{\gamma}^{\infty} k(x, n)\left[\frac{(x-\gamma)^{\left(1-\frac{\alpha-\beta}{2}\right) / q}}{(n-\eta)^{\left(1-\frac{\alpha-\beta}{2}\right) / p}} f(x)\right]\left[\frac{(n-\gamma)^{\left(1-\frac{\alpha-\beta}{2}\right) / p}}{(x-\gamma)^{\left(1-\frac{\alpha-\beta}{2}\right) / q}}\right] d x\right\}^{p}
\end{aligned}
$$




$$
\begin{aligned}
\geq & \int_{\gamma}^{\infty} k(x, n) \frac{(x-\gamma)^{\left(1-\frac{\alpha-\beta}{2}\right)(p-1)}}{(n-\eta)^{1-\frac{\alpha-\beta}{2}}} f^{p}(x) d x \\
& \times\left\{\int_{\gamma}^{\infty} k(x, n) \frac{(n-\eta)^{\left(1-\frac{\alpha-\beta}{2}\right)(q-1)}}{(x-\gamma)^{1-\frac{\alpha-\beta}{2}}} d x\right\}^{p-1} \\
= & \left\{\omega(n)(n-\eta)^{q\left(1-\frac{\alpha-\beta}{2}\right)-1}\right\}^{p-1} \int_{\gamma}^{\infty} k(x, n) \frac{(x-\gamma)^{\left(1-\frac{\alpha-\beta}{2}\right)(p-1)}}{(n-\eta)^{1-\frac{\alpha-\beta}{2}}} f^{p}(x) d x \\
= & \left(\frac{4}{\alpha+\beta}\right)^{p-1}(n-\eta)^{1-\frac{p(\alpha-\beta)}{2}} \int_{\gamma}^{\infty} k(x, n) \frac{(x-\gamma)^{\left(1-\frac{\alpha-\beta}{2}\right)(p-1)}}{(n-\eta)^{1-\frac{\alpha-\beta}{2}}} f^{p}(x) d x .
\end{aligned}
$$

Then by the Lebesgue term by term integration theorem (cf. [20]), we have

$$
\begin{aligned}
J & \geq\left(\frac{4}{\alpha+\beta}\right)^{\frac{1}{q}}\left\{\sum_{n=1}^{\infty} \int_{\gamma}^{\infty} k(x, n) \frac{(x-\gamma)^{\left(1-\frac{\alpha-\beta}{2}\right)(p-1)}}{(n-\eta)^{1-\frac{\alpha-\beta}{2}}} f^{p}(x) d x\right\}^{\frac{1}{p}} \\
& =\left(\frac{4}{\alpha+\beta}\right)^{\frac{1}{q}}\left\{\int_{\gamma}^{\infty} \sum_{n=1}^{\infty} k(x, n) \frac{(x-\gamma)^{\left(1-\frac{\alpha-\beta}{2}\right)(p-1)}}{(n-\eta)^{1-\frac{\alpha-\beta}{2}}} f^{p}(x) d x\right\}^{\frac{1}{p}} \\
& =\left(\frac{4}{\alpha+\beta}\right)^{\frac{1}{q}}\left\{\int_{\gamma}^{\infty} \varpi(x)(x-\gamma)^{p\left(1-\frac{\alpha-\beta}{2}\right)-1} f^{p}(x) d x\right\}^{\frac{1}{p}},
\end{aligned}
$$

and then (14) follows. By the reverse Hölder inequality, for $q<0$, we have

$$
\begin{aligned}
{\left[\sum_{n=1}^{\infty} k(x, n) a_{n}\right]^{q} } & =\left\{\sum_{n=1}^{\infty} k(x, n)\left[\frac{(x-\gamma)^{\left(1-\frac{\alpha-\beta}{2}\right) / q}}{(n-\eta)^{\left(1-\frac{\alpha-\beta}{2}\right) / p}}\right]\left[\frac{(n-\eta)^{\left(1-\frac{\alpha-\beta}{2}\right) / p} a_{n}}{(x-\gamma)^{\left(1-\frac{\alpha-\beta}{2}\right) / q}}\right]\right\}^{q} \\
& \leq\left\{\sum_{n=1}^{\infty} k(x, n) \frac{(x-\gamma)^{\left(1-\frac{\alpha-\beta}{2}\right)(p-1)}}{(n-\eta)^{1-\frac{\alpha-\beta}{2}}}\right\}^{q-1} \sum_{n=1}^{\infty} k(x, n) \frac{(n-\eta)^{\left(1-\frac{\alpha-\beta}{2}\right)(q-1)}}{(x-\gamma)^{1-\frac{\alpha-\beta}{2}}} a_{n}^{q} \\
& =\frac{[\varpi(x)]^{q-1}}{(x-\gamma)^{\frac{q(\alpha-\beta)}{2}}-1} \sum_{n=1}^{\infty} k(x, n) \frac{(n-\eta)^{\left(1-\frac{\alpha-\beta}{2}\right)(q-1)}}{(x-\gamma)^{1-\frac{\alpha-\beta}{2}}} a_{n}^{q} .
\end{aligned}
$$

By the Lebesgue term by term integration theorem, we have

$$
\begin{aligned}
L_{1} & \geq\left\{\int_{\gamma}^{\infty} \sum_{n=1}^{\infty} k(x, n) \frac{(n-\eta)^{\left(1-\frac{\alpha-\beta}{2}\right)(q-1)}}{(x-\gamma)^{1-\frac{\alpha-\beta}{2}}} a_{n}^{q} d x\right\}^{\frac{1}{q}} \\
& =\left\{\sum_{n=1}^{\infty} \int_{\gamma}^{\infty} k(x, n) \frac{(n-\eta)^{\left(1-\frac{\alpha-\beta}{2}\right)(q-1)}}{(x-\gamma)^{1-\frac{\alpha-\beta}{2}}} a_{n}^{q} d x\right\}^{\frac{1}{q}} \\
& =\left\{\sum_{n=1}^{\infty} \omega(n)(n-\eta)^{q\left(1-\frac{\alpha-\beta}{2}\right)-1} a_{n}^{q}\right\}^{\frac{1}{q}},
\end{aligned}
$$

and in view of (11), inequality (15) follows. For $p<0$, by the same way we still have (14) and (15).

Lemma 4 Let the assumptions of Lemma 2 be fulfilled and additionally, let $0<p<1, \frac{1}{p}+$ $\frac{1}{q}=1,0<\varepsilon<\frac{p}{2}(\alpha+\beta)$. Setting $\tilde{f}(x)=(x-\gamma)^{\frac{\alpha-\beta}{2}+\frac{\varepsilon}{p}-1}, x \in(\gamma, \gamma+1) ; \tilde{f}(x)=0, x \in[\gamma+1, \infty)$, 
Yang and Chen Journal of Inequalities and Applications 2014, 2014:96

Page 8 of 13

http://www.journalofinequalitiesandapplications.com/content/2014/1/96

and $\widetilde{a}_{n}=(n-\eta)^{\frac{\alpha-\beta}{2}-\frac{\varepsilon}{q}-1}, n \in \mathbf{N}$, then we have

$$
\begin{aligned}
\widetilde{I} & :=\sum_{n=1}^{\infty} \widetilde{a}_{n} \int_{\gamma}^{\infty} \frac{(\min \{1,(x-\gamma)(n-\eta)\})^{\beta}}{(\max \{1,(x-\gamma)(n-\eta)\})^{\alpha}} \widetilde{f}(x) d x \\
& <\frac{1}{\varepsilon} \frac{(\alpha+\beta)}{\left(\frac{\alpha+\beta}{2}\right)^{2}-\left(\frac{\varepsilon}{p}\right)^{2}}\left[\frac{\varepsilon}{(1-\eta)^{\varepsilon+1}}+\frac{1}{(1-\eta)^{\varepsilon}}\right], \\
\widetilde{H} & :=\left\{\int_{\gamma}^{\infty}(x-\gamma)^{p\left(1-\frac{\alpha-\beta}{2}\right)-1} \widetilde{f}^{p}(x) d x\right\}^{\frac{1}{p}}\left\{\sum_{n=1}^{\infty}(n-\eta)^{q\left(1-\frac{\alpha-\beta}{2}\right)-1} \widetilde{a}_{n}^{q}\right\}^{\frac{1}{q}} \\
& >\frac{1}{\varepsilon}(1-\varepsilon O(1))^{\frac{1}{p}}\left\{\frac{\varepsilon}{(1-\eta)^{\varepsilon+1}}+\frac{1}{(1-\eta)^{\varepsilon}}\right\}^{\frac{1}{q}} .
\end{aligned}
$$

Proof We find

$$
\begin{aligned}
\widetilde{I} & =\sum_{n=1}^{\infty}(n-\eta)^{\frac{\alpha-\beta}{2}-\frac{\varepsilon}{q}-1} \int_{\gamma}^{\gamma+1} \frac{(\min \{1,(x-\gamma)(n-\eta)\})^{\beta}}{(\max \{1,(x-\gamma)(n-\eta)\})^{\alpha}}(x-\gamma)^{\frac{\alpha-\beta}{2}+\frac{\varepsilon}{p}-1} d x \\
& <\sum_{n=1}^{\infty}(n-\eta)^{\frac{\alpha-\beta}{2}-\frac{\varepsilon}{q}-1} \int_{\gamma}^{\infty} \frac{(\min \{1,(x-\gamma)(n-\eta)\})^{\beta}}{(\max \{1,(x-\gamma)(n-\eta)\})^{\alpha}}(x-\gamma)^{\frac{\alpha-\beta}{2}+\frac{\varepsilon}{p}-1} d x \\
& =\frac{\alpha+\beta}{\left(\frac{\alpha+\beta}{2}\right)^{2}-\left(\frac{\varepsilon}{p}\right)^{2}}\left[\frac{1}{(1-\eta)^{\varepsilon+1}}+\sum_{n=1}^{\infty} \frac{1}{(n-\eta)^{\varepsilon+1}}\right] \\
& <\frac{\alpha+\beta}{\left(\frac{\alpha+\beta}{2}\right)^{2}-\left(\frac{\varepsilon}{p}\right)^{2}}\left[\frac{1}{(1-\eta)^{\varepsilon+1}}+\int_{1}^{\infty} \frac{d y}{(y-\eta)^{\varepsilon+1}}\right] \\
& =\frac{1}{\varepsilon} \frac{(\alpha+\beta)}{\left(\frac{\alpha+\beta}{2}\right)^{2}-\left(\frac{\varepsilon}{p}\right)^{2}}\left[\frac{\varepsilon}{(1-\eta)^{\varepsilon+1}}+\frac{1}{(1-\eta)^{\varepsilon}}\right],
\end{aligned}
$$

and then (16) is valid. We obtain

$$
\begin{aligned}
\widetilde{H}= & \left\{\int_{\gamma}^{\gamma+1}\left[1-\frac{1}{2}(1-\eta)^{\frac{\alpha+\beta}{2}}(x-\gamma)^{\frac{\alpha+\beta}{2}}\right](x-\gamma)^{\varepsilon-1} d x\right\}^{\frac{1}{p}} \\
& \times\left\{\frac{1}{(1-\eta)^{\varepsilon+1}}+\sum_{n=2}^{\infty} \frac{1}{(n-\eta)^{\varepsilon+1}}\right\}^{\frac{1}{q}} \\
> & \left(\frac{1}{\varepsilon}-O(1)\right)^{\frac{1}{p}}\left\{\frac{1}{(1-\eta)^{\varepsilon+1}}+\int_{1}^{\infty} \frac{d y}{(y-\eta)^{\varepsilon+1}}\right\}^{\frac{1}{q}} \\
= & \frac{1}{\varepsilon}(1-\varepsilon O(1))^{\frac{1}{p}}\left\{\frac{\varepsilon}{(1-\eta)^{\varepsilon+1}}+\frac{1}{(1-\eta)^{\varepsilon}}\right\}^{\frac{1}{q}},
\end{aligned}
$$

and so (17) is valid.

3 Main results

We introduce the functions

$$
\begin{aligned}
& \Phi(x):=(x-\gamma)^{p\left(1-\frac{\alpha-\beta}{2}\right)-1}, \quad \widetilde{\Phi}(x)=(1-\theta(x)) \Phi(x) \quad(x \in(\gamma, \infty)), \\
& \Psi(n):=(n-\eta)^{q\left(1-\frac{\alpha-\beta}{2}\right)-1} \quad(n \in \mathbf{N}),
\end{aligned}
$$


wherefrom $[\Phi(x)]^{1-q}=(x-\gamma)^{q \frac{\alpha-\beta}{2}-1},[\widetilde{\Phi}(x)]^{1-q}=(1-\theta(x))^{1-q}(x-\gamma)^{q \frac{\alpha-\beta}{2}-1}$ and $[\Psi(n)]^{1-p}=$ $(n-\eta)^{p \frac{\alpha-\beta}{2}-1}$.

Theorem 1 If $0<\alpha+\beta \leq 2, \gamma \in \mathbf{R}, \eta \leq 1-\frac{\alpha+\beta}{8}\left(1+\sqrt{3+\frac{4}{\alpha+\beta}}\right), 0<p<1, \frac{1}{p}+\frac{1}{q}=1, f(x), a_{n} \geq$ $0, f \in L_{p, \widetilde{\Phi}}(\gamma, \infty), a=\left\{a_{n}\right\}_{n=1}^{\infty} \in l_{q, \Psi},\|f\|_{p, \widetilde{\Phi}}>0$ and $\|a\|_{q, \Psi}>0$, then we have the following equivalent inequalities:

$$
\begin{aligned}
I & :=\sum_{n=1}^{\infty} a_{n} \int_{\gamma}^{\infty} \frac{(\min \{1,(x-\gamma)(n-\eta)\})^{\beta}}{(\max \{1,(x-\gamma)(n-\eta)\})^{\alpha}} f(x) d x \\
& =\int_{\gamma}^{\infty} f(x) \sum_{n=1}^{\infty} \frac{(\min \{1,(x-\gamma)(n-\eta)\})^{\beta} a_{n}}{(\max \{1,(x-\gamma)(n-\eta)\})^{\alpha}} d x>\frac{4}{\alpha+\beta}\|f\|_{p, \widetilde{\Phi}}\|a\|_{q, \Psi}, \\
J & =\left\{\sum_{n=1}^{\infty}[\Psi(n)]^{1-p}\left[\int_{\gamma}^{\infty} \frac{(\min \{1,(x-\gamma)(n-\eta)\})^{\beta} f(x)}{(\max \{1,(x-\gamma)(n-\eta)\})^{\alpha}} d x\right]^{p}\right\}^{\frac{1}{p}}>\frac{4}{\alpha+\beta}\|f\|_{p, \widetilde{\Phi}}, \\
L & :=\left\{\int_{\gamma}^{\infty}[\widetilde{\Phi}(x)]^{1-q}\left[\sum_{n=1}^{\infty} \frac{(\min \{1,(x-\gamma)(n-\eta)\})^{\beta} a_{n}}{(\max \{1,(x-\gamma)(n-\eta)\})^{\alpha}}\right]^{q} d x\right\}^{\frac{1}{q}}>\frac{4}{\alpha+\beta}\|a\|_{q, \Psi},
\end{aligned}
$$

where the constant $\frac{4}{\alpha+\beta}$ is the best possible in the above inequalities.

Proof The two expressions for $I$ in (18) follow from Lebesgue's term by term integration theorem. By (14) and (11), we have (19). By the reverse Hölder inequality, we have

$$
I=\sum_{n=1}^{\infty}\left[\Psi^{\frac{-1}{q}}(n) \int_{\gamma}^{\infty} \frac{(\min \{1,(x-\gamma)(n-\eta)\})^{\beta} f(x)}{(\max \{1,(x-\gamma)(n-\eta)\})^{\alpha}} d x\right]\left[\Psi^{\frac{1}{q}}(n) a_{n}\right] \geq J\|a\|_{q, \Psi} .
$$

Then by (19), we have (18). On the other-hand, assume that (18) is valid. Setting

$$
a_{n}:=[\Psi(n)]^{1-p}\left[\int_{\gamma}^{\infty} \frac{(\min \{1,(x-\gamma)(n-\eta)\})^{\beta} f(x)}{(\max \{1,(x-\gamma)(n-\eta)\})^{\alpha}} d x\right]^{p-1}, \quad n \in \mathbf{N},
$$

it follows that $J^{p-1}=\|a\|_{q, \Psi}$. By (14), we find $J>0$. If $J=\infty$, then (19) is trivially valid; if $J<\infty$, then by (18), we have

$$
\|a\|_{q, \Psi}^{q}=J^{q(p-1)}=J^{p}=I>\frac{4}{\alpha+\beta}\|f\|_{p, \widetilde{\Phi}}\|a\|_{q, \Psi},
$$

therefore $\|a\|_{q, \Psi}^{q-1}=J>\frac{4}{\alpha+\beta}\|f\|_{p, \widetilde{\Phi}}$, that is, (19) is equivalent to (18). On the other-hand, by (11) we have $[\varpi(x)]^{1-q}>(1-\theta(x))^{1-q}\left(\frac{4}{\alpha+\beta}\right)^{1-q}$. Then in view of (15), we have (20). By the Hölder inequality, we find

$$
I=\int_{\gamma}^{\infty}\left[\widetilde{\Phi}^{\frac{1}{p}}(x) f(x)\right]\left[\widetilde{\Phi}^{\frac{-1}{p}}(x) \sum_{n=1}^{\infty} \frac{(\min \{1,(x-\gamma)(n-\eta)\})^{\beta} a_{n}}{(\max \{1,(x-\gamma)(n-\eta)\})^{\alpha}}\right] d x \geq\|f\|_{p, \widetilde{\Phi} L .}
$$

Then by (20), we have (18). On the other-hand, assume that (18) is valid. Setting

$$
f(x):=[\widetilde{\Phi}(x)]^{1-q}\left[\sum_{n=1}^{\infty} \frac{(\min \{1,(x-\gamma)(n-\eta)\})^{\beta} a_{n}}{(\max \{1,(x-\gamma)(n-\eta)\})^{\alpha}}\right]^{q-1}, \quad x \in(\gamma, \infty),
$$


then $L^{q-1}=\|f\|_{p, \widetilde{\Phi}}$. By (15), we find $L>0$. If $L=\infty$, then (20) is trivially valid; if $L<\infty$, then by (18), we have

$$
\|f\|_{p, \widetilde{\Phi}}^{p}=L^{p(q-1)}=I>\frac{4}{\alpha+\beta}\|f\|_{p, \widetilde{\Phi}}\|a\|_{q, \Psi},
$$

therefore $\|f\|_{p, \widetilde{\Phi}}^{p-1}=L>\frac{4}{\alpha+\beta}\|a\|_{q, \Psi}$, that is, (20) is equivalent to (18). Hence, (18), (19), and (20) are equivalent.

If there exists a positive number $k\left(\geq \frac{4}{\alpha+\beta}\right)$, such that (18) is valid as we replace $\frac{4}{\alpha+\beta}$ with $k$, then in particular, it follows that $\widetilde{I}>k \widetilde{H}$. In view of (16) and (17), we have

$$
\frac{(\alpha+\beta)}{\left(\frac{\alpha+\beta}{2}\right)^{2}-\left(\frac{\varepsilon}{p}\right)^{2}}\left[\frac{\varepsilon}{(1-\eta)^{\varepsilon+1}}+\frac{1}{(1-\eta)^{\varepsilon}}\right]>k(1-\varepsilon O(1))^{\frac{1}{p}}\left\{\frac{\varepsilon}{(1-\eta)^{\varepsilon+1}}+\frac{1}{(1-\eta)^{\varepsilon}}\right\}^{\frac{1}{q}},
$$

and $\frac{4}{\alpha+\beta} \geq k\left(\varepsilon \rightarrow 0^{+}\right)$. Hence $k=\frac{4}{\alpha+\beta}$ is the best value of $(18)$.

By the equivalence of the inequalities, the constant factor $\frac{4}{\alpha+\beta}$ in (19) and (20) is the best possible.

For $p<0$, we have the dual forms of (18), (19), and (20) as follows:

Theorem 2 If $0<\alpha+\beta \leq 2, \gamma \in \mathbf{R}, \eta \leq 1-\frac{\alpha+\beta}{8}\left(1+\sqrt{3+\frac{4}{\alpha+\beta}}\right), p<0, \frac{1}{p}+\frac{1}{q}=1, f(x), a_{n} \geq 0$, $f \in L_{p, \Phi}(\gamma, \infty), a=\left\{a_{n}\right\}_{n=1}^{\infty} \in l_{q, \Psi},\|f\|_{p, \Phi}>0$ and $\|a\|_{q, \Psi}>0$, then we have the following equivalent inequalities:

$$
\begin{aligned}
& \sum_{n=1}^{\infty} a_{n} \int_{\gamma}^{\infty} \frac{(\min \{1,(x-\gamma)(n-\eta)\})^{\beta}}{(\max \{1,(x-\gamma)(n-\eta)\})^{\alpha}} f(x) d x>\frac{4}{\alpha+\beta}\|f\|_{p, \Phi}\|a\|_{q, \Psi}, \\
& \left\{\sum_{n=1}^{\infty}[\Psi(n)]^{1-p}\left[\int_{\gamma}^{\infty} \frac{(\min \{1,(x-\gamma)(n-\eta)\})^{\beta} f(x)}{(\max \{1,(x-\gamma)(n-\eta)\})^{\alpha}} d x\right]^{p}\right\}^{\frac{1}{p}}>\frac{4}{\alpha+\beta}\|f\|_{p, \Phi}, \\
& \left\{\int_{\gamma}^{\infty}[\Phi(x)]^{1-q}\left[\sum_{n=1}^{\infty} \frac{(\min \{1,(x-\gamma)(n-\eta)\})^{\beta} a_{n}}{(\max \{1,(x-\gamma)(n-\eta)\})^{\alpha}}\right]^{q} d x\right\}^{\frac{1}{q}}>\frac{4}{\alpha+\beta}\|a\|_{q, \Psi},
\end{aligned}
$$

where the constant $\frac{4}{\alpha+\beta}$ is the best possible in the above inequalities.

Proof By means of Lemma 3 and the same way, we can prove that (21), (22), and (23) are valid and equivalent. For $0<\varepsilon \frac{|p|}{2}(\alpha+\beta)$, setting $\widetilde{f}(x)$ and $\tilde{a}_{n}$ as Lemma 4 , if there exists a positive number $k\left(\geq \frac{4}{\alpha+\beta}\right)$, such that (21) is valid as we replace $\frac{4}{\alpha+\beta}$ with $k$, then in particular, by (16), it follows that

$$
\begin{aligned}
& \frac{\alpha+\beta}{\left(\frac{\alpha+\beta}{2}\right)^{2}-\left(\frac{\varepsilon}{p}\right)^{2}}\left[\frac{\varepsilon}{(1-\eta)^{\varepsilon+1}}+\frac{1}{(1-\eta)^{\varepsilon}}\right] \\
& >\varepsilon \widetilde{I}>\varepsilon k\left\{\int_{\gamma}^{\gamma+1}(x-\gamma)^{\varepsilon-1} d x\right\}^{\frac{1}{p}}\left\{\sum_{n=1}^{\infty} \frac{1}{(n-\eta)^{\varepsilon+1}}\right\}^{\frac{1}{q}} \\
& >\varepsilon k\left(\frac{1}{\varepsilon}\right)^{\frac{1}{p}}\left\{\int_{1}^{\infty} \frac{d y}{(y-\eta)^{\varepsilon+1}}\right\}^{\frac{1}{q}}=k\left\{\frac{1}{(1-\eta)^{\varepsilon}}\right\}^{\frac{1}{q}},
\end{aligned}
$$


and $\frac{4}{\alpha+\beta} \geq k\left(\varepsilon \rightarrow 0^{+}\right)$. Hence $k=\frac{4}{\alpha+\beta}$ is the best value of (21). By the equivalence of the inequalities, the constant factor $\frac{4}{\alpha+\beta}$ in (21) and (22) is the best possible.

Remark (i) Since we find

$$
\min _{0<\alpha+\beta \leq 2}\left\{1-\frac{\alpha+\beta}{8}\left(1+\sqrt{3+\frac{4}{\alpha+\beta}}\right)\right\}=\frac{3-\sqrt{5}}{4}=0.19^{+}>0,
$$

then for $\eta=\gamma=0$ in (18), we have

$$
\theta_{0}(x)= \begin{cases}\frac{1}{2} x^{\frac{\alpha+\beta}{2}}, & 0<x \leq 1, \\ 1-\frac{1}{2} x^{-\frac{\alpha+\beta}{2}}, & x>1,\end{cases}
$$

and the following inequality:

$$
\begin{aligned}
& \sum_{n=1}^{\infty} a_{n} \int_{0}^{\infty} \frac{(\min \{1, x n\})^{\beta}}{(\max \{1, x n\})^{\alpha}} f(x) d x \\
& \quad>\frac{4}{\alpha+\beta}\left\{\int_{0}^{\infty}\left(1-\theta_{0}(x)\right) x^{p\left(1-\frac{\alpha-\beta}{2}\right)-1} f^{p}(x) d x\right\}^{\frac{1}{p}}\left\{\sum_{n=1}^{\infty} n^{q\left(1-\frac{\alpha-\beta}{2}\right)-1} a_{n}^{q}\right\}^{\frac{1}{q}} .
\end{aligned}
$$

Hence (18) is a more accurate inequality of (24).

(ii) For $\beta=0$ in (18), we have $0<\alpha \leq 2, \gamma \in \mathbf{R}, \eta \leq 1-\frac{\alpha}{8}\left(1+\sqrt{3+\frac{4}{\alpha}}\right)$,

$$
\theta_{1}(x)= \begin{cases}\frac{1}{2}(x-\gamma)^{\frac{\alpha}{2}}, & 0<x-\gamma \leq \frac{1}{1-\eta} \\ 1-\frac{1}{2}(x-\gamma)^{-\frac{\alpha}{2}}, & x-\gamma>\frac{1}{1-\eta}\end{cases}
$$

and the following inequality:

$$
\begin{aligned}
& \sum_{n=1}^{\infty} a_{n} \int_{\gamma}^{\infty} \frac{f(x) d x}{(\max \{1,(x-\gamma)(n-\eta)\})^{\alpha}} \\
& \quad>\frac{4}{\alpha}\left\{\int_{\gamma}^{\infty}\left(1-\theta_{1}(x)\right)(x-\gamma)^{p\left(1-\frac{\alpha}{2}\right)-1} f^{p}(x) d x\right\}^{\frac{1}{p}}\left\{\sum_{n=1}^{\infty}(n-\eta)^{q\left(1-\frac{\alpha}{2}\right)-1} a_{n}^{q}\right\}^{\frac{1}{q}} ;
\end{aligned}
$$

for $\alpha=0$ in (18), we have $0<\beta \leq 2, \gamma \in \mathbf{R}, \eta \leq 1-\frac{\beta}{8}\left(1+\sqrt{3+\frac{4}{\beta}}\right)$,

$$
\theta_{2}(x)= \begin{cases}\frac{1}{2}(x-\gamma)^{\frac{\beta}{2}}, & 0<x-\gamma \leq \frac{1}{1-\eta}, \\ 1-\frac{1}{2}(x-\gamma)^{-\frac{\beta}{2}}, & x-\gamma>\frac{1}{1-\eta}\end{cases}
$$

and the following inequality:

$$
\begin{aligned}
& \sum_{n=1}^{\infty} a_{n} \int_{\gamma}^{\infty}(\min \{1,(x-\gamma)(n-\eta)\})^{\beta} f(x) d x \\
& >\frac{4}{\beta}\left\{\int_{\gamma}^{\infty}\left(1-\theta_{2}(x)\right)(x-\gamma)^{p\left(1+\frac{\beta}{2}\right)-1} f^{p}(x) d x\right\}^{\frac{1}{p}}\left\{\sum_{n=1}^{\infty}(n-\eta)^{q\left(1+\frac{\beta}{2}\right)-1} a_{n}^{q}\right\}^{\frac{1}{q}} ;
\end{aligned}
$$


for $\beta=\alpha=\lambda$ in (18), we have $0<\lambda \leq 1, \gamma \in \mathbf{R}, \eta \leq 1-\frac{\lambda}{4}\left(1+\sqrt{3+\frac{2}{\lambda}}\right)$,

$$
\theta_{3}(x)= \begin{cases}\frac{1}{2}(x-\gamma)^{\lambda}, & 0<x-\gamma \leq \frac{1}{1-\eta} \\ 1-\frac{1}{2}(x-\gamma)^{-\lambda}, & x-\gamma>\frac{1}{1-\eta}\end{cases}
$$

and the following inequality:

$$
\begin{aligned}
& \sum_{n=1}^{\infty} a_{n} \int_{\gamma}^{\infty}\left[\frac{\min \{1,(x-\gamma)(n-\eta)\}}{\max \{1,(x-\gamma)(n-\eta)\}}\right]^{\lambda} f(x) d x \\
& >\frac{2}{\lambda}\left\{\int_{\gamma}^{\infty}\left(1-\theta_{3}(x)\right)(x-\gamma)^{p-1} f^{p}(x) d x\right\}^{\frac{1}{p}}\left\{\sum_{n=1}^{\infty}(n-\eta)^{q-1} a_{n}^{q}\right\}^{\frac{1}{q}} .
\end{aligned}
$$

(iii) Setting $y=\frac{1}{x-\gamma}+\gamma, g(y)=(y-\gamma)^{\alpha-\beta-2} f\left(\frac{1}{y-\gamma}+\gamma\right), \varphi(y)=(y-\gamma)^{p\left(1-\frac{\alpha-\beta}{2}\right)-1}$ and $\tilde{\varphi}(y)=$ $\left(1-\theta\left(\frac{1}{y-\gamma}+\gamma\right)\right) \varphi(y)$ in (18), by simplification, we obtain the following inequality with the homogeneous kernel:

$$
\sum_{n=1}^{\infty} a_{n} \int_{\gamma}^{\infty} \frac{(\min \{y-\gamma, n-\eta\})^{\beta}}{(\max \{y-\gamma, n-\eta\})^{\alpha}} g(y) d y>\frac{4}{\alpha+\beta}\|g\|_{p, \widetilde{\varphi}}\|a\|_{q, \Psi} .
$$

It is evident that (28) is equivalent to (18), and then the same constant factor $\frac{4}{\alpha+\beta}$ in (28) is still the best possible. In the same way, we can find the following inequalities equivalent to (28) with the same best possible constant factor $\frac{4}{\alpha+\beta}$ :

$$
\begin{aligned}
& \left\{\sum_{n=1}^{\infty}[\Psi(n)]^{1-p}\left[\int_{\gamma}^{\infty} \frac{(\min \{y-\gamma, n-\eta\})^{\beta} g(y)}{(\max \{y-\gamma, n-\eta\})^{\alpha}} d y\right]^{p}\right\}^{\frac{1}{p}}>\frac{4}{\alpha+\beta}\|f\|_{p, \widetilde{\varphi}}, \\
& \left\{\int_{\gamma}^{\infty}[\tilde{\varphi}(y)]^{1-q}\left[\sum_{n=1}^{\infty} \frac{(\min \{y-\gamma, n-\eta\})^{\beta} a_{n}}{(\max \{y-\gamma, n-\eta\})^{\alpha}}\right]^{q} d y\right\}^{\frac{1}{q}}>\frac{4}{\alpha+\beta}\|a\|_{q, \Psi} .
\end{aligned}
$$

(iv) Applying the same way in Theorem 2, we still can obtain some particular dual forms as (i) and (ii) and some equivalent inequalities similar to (28), (29), and (30).

\section{Competing interests}

The authors declare that they have no competing interests.

\section{Authors' contributions}

The main idea of this paper was proposed by BY and QC. BY prepared the manuscript initially and performed all the steps of the proofs in this research. All authors read and approved the final manuscript.

\section{Author details}

'Department of Mathematics, Guangdong University of Education, Guangzhou, Guangdong 510303, P.R. China.

${ }^{2}$ Department of Computer Science, Guangdong University of Education, Guangzhou, Guangdong 510303, P.R. China.

\section{Acknowledgements}

This work is supported by the National Natural Science Foundation of China (No. 61370186), 2012 Knowledge Construction Special Foundation Item of Guangdong Institution of Higher Learning College and University (No. 2012KJCX0079), and Guangzhou Science and Technology Plan Item (No. 2013J4100009). 


\section{References}

1. Hardy, GH, Littlewood, JE, Pólya, G: Inequalities. Cambridge University Press, Cambridge (1934)

2. Mitrinović, DS, Pečarić, JE, Fink, AM: Inequalities Involving Functions and Their Integrals and Derivatives. Kluwer Academic, Boston (1991)

3. Yang, B: Hilbert-Type Integral Inequalities. Bentham Science Publishers, Dubai (2009)

4. Yang, B: Discrete Hilbert-Type Inequalities. Bentham Science Publishers, Dubai (2011)

5. Yang, B: On Hilbert's integral inequality. J. Math. Anal. Appl. 220, 778-785 (1998)

6. Yang, B: The Norm of Operator and Hilbert-Type Inequalities. Science Press, Beijing (2009)

7. Yang, B, Brnetić, I, Krnić, M, Pečarić, J: Generalization of Hilbert and Hardy-Hilbert integral inequalities. Math. Inequal Appl. 8(2), 259-272 (2005)

8. Krnić, M, Pečarić, J: Hilbert's inequalities and their reverses. Publ. Math. (Debr.) 67(3-4), 315-331 (2005)

9. Jin, J, Debnath, L: On a Hilbert-type linear series operator and its applications. J. Math. Anal. Appl. 371, 691-704 (2010)

10. Azar, L: On some extensions of Hardy-Hilbert's inequality and applications. J. Inequal. Appl. 2009, Article ID 546829 (2009)

11. Yang, B, Rassias, T: On the way of weight coefficient and research for Hilbert-type inequalities. Math. Inequal. Appl. 6(4), 625-658 (2003)

12. Arpad, B, Choonghong, O: Best constant for certain multilinear integral operator. J. Inequal. Appl. 2006, Article ID $28582(2006)$

13. Kuang, J, Debnath, L: On Hilbert's type inequalities on the weighted Orlicz spaces. Pac. J. Appl. Math. 1(1), 95-103 (2007)

14. Zhong, W: The Hilbert-type integral inequalities with a homogeneous kernel of - $\lambda$-degree. J. Inequal. Appl. 2008 Article ID 917392 (2008)

15. Li, Y, He, B: On inequalities of Hilbert's type. Bull. Aust. Math. Soc. 76(1), 1-13 (2007)

16. Yang, B: A mixed Hilbert-type inequality with a best constant factor. Int. J. Pure Appl. Math. 20(3), 319-328 (2005)

17. Yang, B: A half-discrete Hilbert's inequality. J. Guangdong Univ. Educ. 31(3), 1-7 (2011)

18. Yang, B, Chen, Q: A half-discrete Hilbert-type inequality with a homogeneous kernel and an extension. J. Inequal. Appl. 2011, 124 (2011). doi:10.1186/1029-242X-2011-124

19. Kuang, J: Applied Inequalities. Shangdong Science Technic Press, Jinan (2004)

20. Kuang, J: Introduction to Real Analysis. Hunan Education Press, Changsha (1996)

\subsection{6/1029-242X-2014-96}

Cite this article as: Yang and Chen: A more accurate half-discrete reverse Hilbert-type inequality with a

non-homogeneous kernel. Journal of Inequalities and Applications 2014, 2014:96

\section{Submit your manuscript to a SpringerOpen ${ }^{\circ}$ journal and benefit from:}

- Convenient online submission

Rigorous peer review

- Immediate publication on acceptance

- Open access: articles freely available online

- High visibility within the field

- Retaining the copyright to your article 